\title{
TLR7 GIn11Leu single nucleotide polymorphism and susceptibility to cutaneous melanoma
}

\author{
LISA ELEFANTI $^{1}$, GIORGIA SACCO ${ }^{2}$, CAMILLA STAGNI $^{3}$, MARCO RASTRELLI ${ }^{4}$, \\ CHIARA MENIN ${ }^{1}$, IRENE RUSSO ${ }^{2}$ and MAURO ALAIBAC ${ }^{2}$ \\ ${ }^{1}$ Immunology and Molecular Oncology Unit, Veneto Institute of Oncology, \\ Scientific Institute for Hospitalization, Treatment and Research, Padua I-35128; \\ ${ }^{2}$ Department of Medicine, Dermatology Unit, University of Padua, Padua I-35121; \\ ${ }^{3}$ Department of Surgery, Oncology and Gastroenterology, Oncology and Immunology Unit, University of Padua, \\ Padua I-35100; ${ }^{4}$ Melanoma and Soft Tissue Sarcoma Unit, Veneto Institute of Oncology, \\ Scientific Institute for Hospitalization, Treatment and Research, Padua I-35128, Italy
}

Received February 8, 2015; Accepted February 17, 2016

DOI: $10.3892 / \mathrm{ol} .2016 .4584$

\begin{abstract}
Cutaneous melanoma is a life-threatening skin cancer. Its incidence is rapidly increasing, and early diagnosis is the main factor able to improve its poor prognosis. Toll-like receptors (TLRs) are transmembrane glycoproteins that recognize pathogen- and damage-associated molecular patterns, against which TLRs activate the innate immune response and initiate the adaptive immune response. Genetic variations of these receptors may alter the immune system, and are involved in evolution and susceptibility to various diseases, including cancer. The aim of the present study was to evaluate whether the presence of TLR7 glutamine (Gln) 11 leucine (Leu) polymorphism confers an increased susceptibility to cutaneous melanoma. For that purpose, a case-control study was performed with 182 melanoma cases and 89 controls. To highlight the possible association between the aforementioned polymorphism and the susceptibility to melanoma, 93 cases of single melanoma and 89 cases of multiple primary melanoma (MPM) were compared in the present study. Since the TLR7 gene is localized on the chromosome $\mathrm{X}$, the allelic frequency of the Gln11Leu polymorphism was analyzed separately in males and females. The distribution of allele frequencies between melanoma cases and controls $(\mathrm{P}=0.245)$ and between single melanoma and MPM cases $(\mathrm{P}=0.482)$ was not significant. Therefore, the present results do not suggest an association between TLR7 Gln11Leu polymorphism and susceptibility to cutaneous melanoma. Further studies are required to analyze the influence of other TLR polymorphisms
\end{abstract}

Correspondence to: Professor Mauro Alaibac, Department of Medicine, Dermatology Unit, University of Padua, Via Battisti 206, Padua I-35121, Italy

E-mail: mauro.alaibac@unipd.it

Key words: cutaneous melanoma, TLR7, innate immunity on the susceptibility to malignant melanoma and the involvement of innate immunity in this malignancy.

\section{Introduction}

Cutaneous melanoma is a life-threatening skin tumor whose incidence has increased faster in the last decades than that of any other solid tumor (1). Melanoma has become one of the most frequent types of cancer among Caucasian populations (2), with incidence rates reported to vary between 21.9/100,000 patients/year in the USA and 55.9/100,000 patients/year in Australian males (3). Cutaneous melanoma represents the most common type of tumor among young adults aged 25-29 years, and the second most common neoplasm in those aged $15-29$ years $(4,5)$. Thus, melanoma is currently a significant public health problem in fair-skinned populations worldwide (6). In parallel with the increase in incidence rate, there has also been an increase in the rate of melanoma associated-mortality (2). Distant metastatic melanoma has a 5-year overall survival rate of only 5-10\%, and a median survival of 6-10 months, depending on the site of metastasis (7).

The risk of developing melanoma depends on genetic and environmental factors (1). The major established environmental risk factor for cutaneous malignant melanoma is exposure to solar ultraviolet (UV) light, particularly when intermittent and associated with sunburns (8). Furthermore, childhood and adolescence have been reported to be critical ages for the development of melanoma later in life, since the latent period from initiation of melanoma carcinogenesis to clinical presentation may last decades (1).

Host factors are also important, with the number of nevi being the most powerful predictor of melanoma risk (9). In addition, pigmentary characteristics such as hair, eyes and skin color and Fitzpatrick scale (which aids to determine the sensitivity of the skin to sunburns and its ability to tan), together with family history and actinic damage indicators, also influence the susceptibility to melanoma (10). 
Of all cutaneous melanomas, $\sim 10 \%$ occur in a familial setting with $\geq 2$ close relatives affected, indicating the implication of genes with low prevalence but high penetrance (10). Low-risk or moderate-risk alleles with high prevalence and low penetrance are the most frequent cause of sporadic cases of melanoma (11). Of all primary cutaneous melanomas, $43 \%$ present a mutation in the RAF viral murine sarcoma viral oncogene homolog B gene, but do not exhibit a significant increase in the number of metastatic lesions (12). Mutations that activate mitogen-activated protein kinase signaling pathways are reported to be present in $20 \%$ of cutaneous melanomas (affecting the neuroblastoma RAS viral oncogene homolog) and in $83 \%$ of uveal melanomas [affecting the guanine nucleotide binding protein ( $G$ protein), q polypeptide or the guanine nucleotide binding protein ( $\mathrm{G}$ protein), alpha 11 (Gq class) genes] $(13,14)$.

Significant inflammation with phagocytic infiltration may be observed in several tumors, including cutaneous melanoma, suggesting that the innate immune system may participate in tumor defence (15). Toll-like receptors (TLRs), which have recently emerged as a key component of the innate immune system, are responsible for detecting microbial infections and triggering antimicrobial host defence responses $(16,17)$. However, TLRs have been demonstrated to be important for innate immune response specificity and adaptive immune responses, including maturation of dendritic cells, expression of co-stimulatory molecules and promotion of $\mathrm{T}$ helper (Th)-1 cell-mediated responses through increased production of interleukin (IL)-12 (18).

TLRs serve as signaling molecules that recognize exogenous pathogen-associated molecular patterns (PAMPs) and endogenous damage-associated molecular patterns (DAMPs) (19). TLRs are a family of type I integral membrane glycoproteins, and based on its considerable homology in the cytoplasmic region, TLRs are also considered members of a larger superfamily that includes IL-1 receptors (IL-1Rs) (20). The human TLR family consists of 13 members, which are structurally characterized by the presence of a leucine-rich repeat domain in their extracellular region and a Toll/IL-1R domain in their intracellular region (21).

Several members of the TLR family are localized on the cell surface (including TLR1, TLR2, TLR4 and TLR6), whereas other members (such as TLR3, TLR7, TLR8 and TLR9) are localized in endosomal membranes inside the cell $(22,23)$. Through the adaptor molecule myeloid differentiation primary response protein 88 (MyD88), TLRs activate signaling pathways that are responsible for the induction of interferon (IFN) regulatory factor, activator protein-1 and nuclear factor kappa-light-chain-enhancer of activated B cells $(20,24,25)$. The genes induced by the activation of TLRs produce several inflammatory cytokines, including tumor necrosis factor (TNF)-alpha, IFN-1, IL-6, IL-1 and granulocyte-colony stimulating factor (21). Different TLRs have been associated with the pathogenesis of several inflammatory and autoimmune skin diseases (26), and have gained interest in cancer research due to their role in tumor development and progression (18). Melanoma cells have been reported to express TLRs 2, 3, 4, 7, 8 and 9 (27), and it has been demonstrated that tumor cells expressing TLRs may be able to evade immune surveillance processes, thus promoting
Table I. Characteristics of patients with cutaneous melanoma included in the present study.

\begin{tabular}{lc}
\hline Characteristics & No. of patients \\
\hline Total & 182 \\
Gender & \\
Male & 85 \\
Female & 99 \\
Median age, years (range) & $43(15-75)$ \\
Histological type & \\
In situ & 15 \\
Superficial spreading & 138 \\
Acral & 5 \\
Nodular & 17 \\
Lentigo maligna & 2 \\
Undefined & 5 \\
Tumor thickness, mm & \\
0 & 134 \\
$1.01-2.00$ & 29 \\
$2.01-4.00$ & 14 \\
$>4.00$ & 5 \\
Clark's level & \\
Not determined-I & \\
II & 22 \\
III & 45 \\
V & 66 \\
Multiple primary melanomas, no. & 46 \\
2 & 3 \\
3 & 89 \\
$\geq 4$ & 50 \\
\hline
\end{tabular}

tumor development (28). In this regard, the activation of TLRs in tumor cells promotes tumor cell proliferation and resistance to apoptosis, and enhances tumor cell invasion and metastasis by regulating metalloproteinases and integrins (29). Furthermore, the activation of TLR signaling in tumor cells induces the synthesis of pro-inflammatory factors and immunosuppressive molecules, which enhance the resistance of tumor cells to cytotoxic lymphocyte attack, thus leading to immune evasion (29). This indicates that targeting tumor TLR signaling pathways may be a suitable therapeutic approach for the treatment of cancer (29). In this regard, topical imiquimod has been demonstrated to be an efficacious TLR-modulating anti-skin cancer agent (30). In addition, other topical agents, including nicotinamide, all-trans retinoic acid, adapalene, zinc, and sodium tosylchloramide, have also been demonstrated to exert their anti-cancer action partially through TLRs (30).

TLR genes are polymorphic (31). While this may be advantageous at the population level, there may be unfavorable outcomes for those individuals who harbour certain genotypes (31). The two main risk factors for developing melanoma are UV exposure and genetic predisposition (32). The identification of possible elements of susceptibility to 
Table II. Genotype frequencies of the single nucleotide polymorphism rs179008 (Gln11Leu) in the Toll-like receptor 7 gene.

Patients

\begin{tabular}{|c|c|c|c|c|c|c|c|c|}
\hline \multirow[b]{3}{*}{ rs179008 } & \multirow{2}{*}{\multicolumn{2}{|c|}{ Controls }} & \\
\hline & & & \multicolumn{2}{|c|}{ Total } & \multicolumn{2}{|c|}{ Single melanoma } & \multicolumn{2}{|c|}{$\begin{array}{l}\text { Multiple primary } \\
\text { melanoma }\end{array}$} \\
\hline & No. & $\%$ & No. & $\%$ & No. & $\%$ & No. & $\%$ \\
\hline \multicolumn{9}{|l|}{ Females } \\
\hline Total & 37 & 100.00 & 97 & 100.00 & 57 & 100.00 & 40 & 100.00 \\
\hline AA & 24 & 64.86 & 57 & 58.76 & 33 & 57.89 & 24 & 60.00 \\
\hline AT & 12 & 32.43 & 32 & 32.99 & 19 & 33.33 & 13 & 32.50 \\
\hline $\mathrm{TT}$ & 1 & 2.70 & 8 & 8.25 & 5 & 8.77 & 3 & 7.50 \\
\hline \multicolumn{9}{|l|}{ Males } \\
\hline Total & 52 & 100.00 & 85 & 100.00 & 36 & 100.00 & 49 & 100.00 \\
\hline $\mathrm{A}$ & 43 & 82.69 & 66 & 77.65 & 26 & 72.22 & 40 & 81.63 \\
\hline $\mathrm{T}$ & 9 & 17.31 & 19 & 22.35 & 10 & 27.78 & 9 & 18.37 \\
\hline \multicolumn{9}{|l|}{ Alleles } \\
\hline Total & 126 & 100.00 & 279 & 100.00 & 150 & 100.00 & 129 & 100.00 \\
\hline A & 103 & 81.75 & 212 & 75.99 & 111 & 74.00 & 101 & 78.29 \\
\hline $\mathrm{T}$ & 23 & 18.25 & 67 & 24.01 & 39 & 26.00 & 28 & 21.71 \\
\hline
\end{tabular}

The sum of normal and polymorphic allele frequencies was used to obtain data regarding alleles.

melanoma may be useful to identify a new targeted screening and diagnostic approach for skin cancer (31). Polymorphisms of TLRs may affect the normal production of pro- and anti-inflammatory cytokines, thus modulating the risk of infection, chronic inflammation and cancer (31). Currently, several TLR polymorphisms are being studied to assess their implications in terms of susceptibility, severity and prognosis towards malignancies (31).

The present study aimed to analyze the glutamine (Gln) 11 leucine (Leu) polymorphism of TLR7 to assess whether carriers of this variant are predisposed to cutaneous melanoma. The TLR7 gene is located on the chromosome X and contains three exons (33). In the aforementioned polymorphism, the Leu variant encoded by the $\mathrm{T}$ allele of the non-synonymous single nucleotide polymorphism (SNP) rs179008 is located within the exon 3 of TLR7, and leads to the replacement of the wild-type allele A encoding Gln at the codon 11 for Leu in the protein (Gln11Leu). The rationale of the preset study is based on the fact that imiquimod, a TLR7 agonist, has been demonstrated to be effective in the treatment of lentigo maligna (the in situ variant of lentigo maligna melanoma) (34), which suggests the participation of TLR7 in the onset and progression of cutaneous melanoma. Therefore, polymorphisms of this receptor may be responsible for a greater or lesser susceptibility to melanoma.

\section{Materials and methods}

Patients. In the present study, a case-control study was performed at the Veneto Institute of Oncology (Padua, Italy) using 182 cases of histologically confirmed cutaneous melanoma [staged according to the 2009 American Joint
Committee on Cancer Melanoma Staging and Classification (35)] and 89 controls from healthy blood donors. To highlight the potential association between the Gln11Leu TLR7 polymorphism and the risk of melanoma, 89 cases of multiple primary melanoma (MPM) and 93 cases of single diagnosis of melanoma (as defined by $\geq 5$ years of follow-up without successive melanoma), were included among the group of patients in the present study. Data about gender, age at diagnosis, histopathological characteristics and number of primary melanomas for the MPM cases are reported in Table I. All the participants were Caucasian and provided written informed consent for participation in the study. The present study was approved by the Ethics Committee of Veneto Institute of Oncology (Padua, Italy).

Quantitative polymerase chain reaction $(q P C R)$. DNA was extracted from peripheral blood samples, and genotyping of the TLR7 SNP rs179008 was performed by allelic discrimination using the $\operatorname{TaqMan}^{\circledR}$ SNP Genotyping Assay (catalogue number C_2259574_10; Applied Biosystems; Thermo Fisher Scientific, Inc., Waltham, MA, USA). The sequences of the primers and TaqMan minor groove binder probes used were as follows: Foward, 5'-CTTTCAGGTGTTTCCAATGTG GAC-3' and reverse, 5'-CCCCAAGGAGTTTGGAAATTAGGAT-3' for primer; and forward, 5'-TGAAGAGACAAATTC-3' and reverse, 5'-ACTGAAGAGACTAATTC-3' for TaqMan probe. PCR was conducted in a StepOnePlus ${ }^{\mathrm{TM}}$ Real-Time PCR System (Applied Biosystems; Thermo Fisher Scientific, Inc.), according to the manufacturer's protocol, under the following conditions: $50^{\circ} \mathrm{C}$ for $2 \mathrm{~min}, 95^{\circ} \mathrm{C}$ for $10 \mathrm{~min}$, followed by 40 cycles at $95^{\circ} \mathrm{C}$ for $15 \mathrm{sec}$ and $60^{\circ} \mathrm{C}$ for $1 \mathrm{~min}$. 
Statistical analysis. The genotypes obtained were analyzed using the Fisher's exact test, which was used to analyze associations between categorical variables. The allele frequency of rs179008 TLR7, which is an X-linked gene, was analyzed separately for males and females, as well as overall in the melanoma patient and control groups. The allelic frequency of the subgroup of MPM cases was compared with that of the single melanoma cases.

\section{Results and Discussion}

In the present study, no significant differences were observed in the distribution of allele frequencies between melanoma cases and controls $(\mathrm{P}=0.245)$, or between MPM vs. single melanoma cases $(\mathrm{P}=0.482)$. Therefore, the present results do not suggest the involvement of the Gln11Leu polymorphism of TLR7 in the susceptibility to malignant melanoma (Table II).

The TLR family comprises membrane glycoproteins that recognize specific PAMPs (16). TLRs are key elements to detect microbial infections and to initiate the immune response against them (16). TLRs activation directly initiates inflammatory and innate immune responses, and triggers the antigen-specific adaptive immune response $(16,17)$. TLRs are also capable of recognizing molecular patterns that are typical of endogenous cellular damage (DAMPs), and are thus implicated in processes of cellular proliferation and survival, apoptosis, angiogenesis and tissue remodelling (36). TLRs are expressed by immune cells and other cellular types, including cancer cells $(37,38)$. Significant quantities of DAMPs are released in the tumor microenviroment by damaged epithelial cells and necrotic tumor cells (28). These molecules activate specific TLRs, leading to further chronic inflammation, which is one of the main cancer-promoting factors (28). The expression of TLRs in the tumor microenviroment directly influences tumor development and its interactions with the immune system (38). The activation of TLRs upregulates pro-oncogenic pathways via the induction of nitric oxide synthase and cyclooxygenase 2 , which regulate the expression of TLRs and promote a positive feedback mechanism, thus leading to the progression of cancer and the development of more aggressive neoplastic phenotypes (38). This mechanism involves tumor cells, stroma and immune system cells (39). However, TLR ligands may also exert an anti-cancer effect, as demonstrated by studies on TLR9 agonists and TLR7/8 agonists such as imiquimod (40-42). Furthermore, Wang et al (43) investigated the action of a dual TLR7/8 agonist in tumor-bearing mice, and demonstrated that subcutaneous administration of the above TLR7/8 agonist effectively prevented lung metastasis by inducing Th1-type immune responses and potent antitumor activity in mice via TLR7 and MyD88-dependent pathways (43). In addition, the TLR7 agonist imiquimod has been successfully applied in the treatment of actinic keratosis, basal cell carcinoma, Bowen's disease and lentigo maligna $(44,45)$.

Genetic host factors are major determinants of disease risk for several types of cancer (31). Genetic variation in immune-regulating components such as TLRs may lead to differences in immune response and susceptibility to different diseases, including inflammatory-autoimmune disorders and cancer (36). SNPs are one of the most common genetic variants that may affect human DNA (45). SNPS may lead to an amino acid substitution in the protein encoded by a particular gene, thus altering its function (46). The genes coding for TLRs have been observed to be polymorphic (34). The impact of various TLRs polymorphisms on the severity and prognosis of numerous autoimmune-inflammatory diseases and several types of cancer is currently under investigation (36). Furthermore, the impact of TLRs polymorphisms on survival and susceptibility to melanoma has been previously analyzed (46). Gast et al (47) analyzed SNPs in different TLRs (1-6, 9 and 10), and demonstrated an association between the polymorphism rs4986790 (D299G) of TLR4 and increased survival in metastatic melanoma. However, the authors did not observe any significant correlation between these SNPs and the incidence of melanoma (47). In the present study, the Gln11Leu polymorphism of TLR7, which is localized on the chromosome X 22.2 at the ATG start codon of exon 3., was analyzed. The TLR7 Gln11Leu polymorphism is a functional non-synonymous polymorphism that consists in the replacement of an adenine by a thymine $(\mathrm{A} / \mathrm{T})$ in the DNA, resulting in the substitution of Gln for Leu in position 11 of the polypeptide chain of TLR7 (48). This polymorphism was selected based on previous studies suggesting the participation of TLR7 in the pathogenesis and progression of melanoma (27). TLR7 expression in melanoma cells was demonstrated both in vitro and in vivo by Saint-Jean et al (27), who performed a study on melanoma cell lines and lymph nodes invaded by melanoma metastasis. Furthermore, imiquimod has been successfully used to topically treat facial lentigo maligna, thus providing a good therapeutic alternative to the often disfiguring surgical excision (34). Imiquimod is a synthetic TLR7 agonist that has been used in the topical treatment of warts, actinic keratoses, Bowen's disease, basal cell carcinoma, extramammary Paget's disease and lentigo maligna (44). Although the exact mechanism of action of this drug remains unknown, numerous clinical studies have demonstrated that imiquimod induces the expression of several cytokines, including IFN-alpha, IL-12 and TNF-alpha, which mediate its antiviral and anticancer (pro-apoptotic, anti-invasive and anti-angiogenic) effects (46). These results were confirmed by Aspord et al (41), who demonstrated the inhibition of melanoma development by imiquimod on humanized melanoma-bearing mice, thus suggesting the potential clinical use of this drug in the treatment of metastatic melanoma. Currently, imiquimod is only available for topical application, however, we hypothesize that the future utilization of this TLR7 agonist in the systemic therapy of metastatic melanoma may be possible, since the therapies currently available for metastatic melanoma are largely unsatisfactory.

In order to evaluate whether the presence of the Gln11Leu polymorphism in the TLR7 gene may confer an increased susceptibility to cutaneous melanoma, the present authors conducted a case-control study, in which the distribution of frequencies of wild-type TLR7 and mutant TLR7 carrying the Gln11Leu polymorphism was analyzed in healthy subjects and patients with melanoma. If the frequency of this polymorphism in patients with melanoma had been higher than in healthy subjects, and/or if this variant had been more frequently present in cases of MPM compared with cases of single melanoma, the Gln11Leu polymorphism of TLR7 would have been considered as a cofactor in the induction of cutaneous melanoma. However, the results of the present study do not suggest the involvement of this SNP in the susceptibility to malignant melanoma, as no 
significant differences were observed in the distribution of allele frequencies between melanoma cases and healthy controls, nor between MPM cases and single melanoma cases. However, the present study exhibits certain limitations, mainly due to the small number of samples. Furthermore, other genetic variants of TLR7 remain largely unexplored to date. Therefore, other polymorphisms of TLR7 may influence the risk of melanoma.

In conclusion, the immune system is important in the defence against exogenous pathogens and in tumor surveillance. The influence of various components of the immune system on tumor onset and progression has been demonstrated, and numerous studies have contributed to identify and clarify the mechanisms involved. The influence of TLRs polymorphisms on the susceptibility to cutaneous melanoma requires further studies to confirm these preliminary observations and to identify potential diagnostic and therapeutic applications of these polymorphisms.

\section{References}

1. Eggermont AM, Spatz A and Robert C: Cutaneous melanoma. Lancet 383: 816-827, 2014.

2. Rastrelli M, Alaibac M, Stramare R, Chiarion Sileni V, Montesco MC, Vecchiato A, Campana LG and Rossi CR: Melanoma m (zero): Diagnosis and therapy. ISRN Dermatol 2013: 616170, 2013

3. Gandini S, Autier P and Boniol M: Reviews on sun exposure and artificial light and melanoma. Prog Biophys Mol Biol 107: 362-366, 2011

4. Christenson LJ, Borrowman TA, Vachon CM, Tollefson MM, Otley CC, Weaver AL and Roenigk RK: Incidence of basal cell and squamous cell carcinomas in a population younger than 40 years. JAMA 294: 681-690, 2005.

5. Birch-Johansen F, Jensen A, Mortensen L, Olesen AB and Kjær SK: Trends in the incidence of nonmelanoma skin cancer in Denmark 1978-2007: Rapid incidence increase among young Danish women. Int J Cancer 127: 2190-2198, 2010.

6. Rastrelli M, Tropea S, Pigozzo J, Bezzon E, Campana LG, Stramare R, Alaibac M and Rossi CR: Melanoma m1: Diagnosis and therapy. In Vivo 28: 273-285, 2014.

7. Panasiti V, Curzio M, Roberti V, Lieto P, Devirgiliis V, Gobbi S, Naspi A, Coppola R, Lopez T, di Meo N, et al: Metastatic volume: An old oncologic concept and a new prognostic factor for stage IV melanoma patients. Dermatology 227: 55-61, 2013.

8. Gandini S, Sera F, Cattaruzza MS, Pasquini P, Picconi O, Boyle P and Melchi CF: Meta-analysis of risk factors for cutaneous melanoma: II. Sun exposure. Eur J Cancer 41: 45-60, 2005.

9. Gandini S, Sera F, Cattaruzza MS, Pasquini P, Abeni D, Boyle P and Melchi CF: Meta-analysis of risk factors for cutaneous melanoma: I. Common and atypical naevi. Eur J Cancer 41: 28-44, 2005.

10. Gandini S, Sera F, Cattaruzza MS, Pasquini P, Zanetti R, Masini C, Boyle P and Melchi CF: Meta-analysis of risk factors for cutaneous melanoma: III. Family history, actinic damage and phenotypic factors. Eur J Cancer 41: 2040-2059, 2005

11. Tsao H, Chin L, Garraway LA and Fisher DE: Melanoma: From mutations to medicine. Genes Dev 26: 1131-1155, 2012.

12. Colombino M, Capone M, Lissia A, Cossu A, Rubino C De Giorgi V, Massi D, Fonsatti E, Staibano S, Nappi O, et al: BRAF/NRAS mutation frequencies among primary tumors and metastases in patients with melanoma. J Clin Oncol 30: 2522-2529, 2012.

13. Platz A, Egyhazi S, Ringborg U and Hansson J: Human cutaneous melanoma; a review of NRAS and BRAF mutation frequencies in relation to histogenetic subclass and body site. Mol Oncol 1: 395-405, 2008.

14. Van Raamsdonk CD, Bezrookove V, Green G, Bauer J, Gaugler L, O'Brien JM, Simpson EM, Barsh GS and Bastian BC: Frequent somatic mutations of GNAQ in uveal melanoma and blue naevi. Nature 457: 599-602, 2009.

15. Navarini-Meury AA and Conrad C: Melanoma and innate immunity - Active inflammation or just erroneous attraction? Melanoma as the source of leukocyte-attracting chemokines. Semin Cancer Biol 19: 84-91, 2009.
16. Iwasaki A and Medzhitov R: Toll-like receptor control of the adaptive immune responses. Nat Immunol 5: 987-995, 2004.

17. Medzhitov R: Toll-like receptors and innate immunity. Nat Rev Immunol 1: 135-145, 2001.

18. Burns EM and Yusuf N: Toll-like receptors and skin cancer. Front Immunol 5: 135, 2014.

19. Akira S and Hemmi H: Recognition of pathogen associated molecular patterns by TLR family. Immunol Lett 85: 85-95, 2003.

20. Akira S and Takeda K: Toll-like receptor signaling. Nat Rev Immunol 4: 499-511, 2004.

21. Eiró N, Ovies C, Fernandez-Garcia B, Álvarez-Cuesta CC, González L, González LO and Vizoso FJ: Expression of TLR 3, 4, 7 and 9 in cutaneous malignant melanoma: Relationship with clinicopathological characteristics and prognosis. Arch Dermatol Res 305: 59-67, 2013.

22. Miyake K: Innate immune sensing of pathogens and danger signals by cell surface Toll-like receptors. Semin Immunol 19: 3-10, 2007.

23. Häcker H, Mischak H, Miethke T, Liptay S, Schmid R, Sparwasser T, Heeg K, Lipford GB and Wagner H: CpG-DNA-specific activation of antigen-presenting cells requires stress kinase activity and is preceded by non-specific endocytosis and endosomal maturation. EMBO J 17: 6230-6240, 1998.

24. Seki E and Brenner DA: Toll-like receptors and adaptor molecules in liver disease: Update. Hepatology 48: 322-335, 2008.

25. Moynagh PN: TLR signaling and activation of IRFs: Revisiting old friends from the NF-kappaB pathway. Trends Immunol 26: 469-476, 2005.

26. Ermertcan AT, Öztürk F and Gündüz K: Toll-like receptors and skin. J Eur Acad Dermatol Venereol 25: 997-1006, 2011.

27. Saint-Jean M, Knol AC, Nguyen JM, Khammari A and Dréno B: TLR expression in human melanoma cells. Eur J Dermatol 21: 899-905, 2011.

28. Sato Y, Goto Y, Narita N and Hoon DS: Cancer cells expressing toll-like receptors and the tumor microenvironment. Cancer Microenviron 2 (Suppl 1): 205-214, 2009.

29. Huang B, Zhao J, Unkeless JC, Feng ZH and Xiong H: TLR signaling by tumor and immune cells: A double-edged sword. Oncogene 27: 218-224, 2008.

30. Valins W, Amini S and Berman B: The expression of Toll-like receptors in dermatological diseases and the therapeutic effect of current and newer topical Toll-like receptor modulators. J Clin Aesthet Dermatol 3: 20-29, 2010.

31. El-Omar EM, Ng MT and Hold GL: Polymorphisms in Toll-like receptor genes and risk of cancer. Oncogene 27: 244-252, 2008.

32. Maire C, Vercambre-Darras S and Desmedt E: Diagnosis of melanoma. Rev Prat 64: 61-68, 2014 (In French).

33. Bordignon M, Bargagli E, Agostini C, Cinetto F, Baldo V, Alaibac M and Rottoli P: TLR7 Gln11Leu single nucleotide polymorphism in patients with sarcoidosis. Sarcoidosis Vasc Diffuse Lung Dis 30: 157-161, 2013.

34. Wong JG, Toole JW, Demers AA, Musto G and Wiseman MC: Topical 5\% imiquimod in the treatment of lentigo maligna. J Cutan Med Surg 16: 245-249, 2012.

35. Balch CM, Gershenwald JE, Soong SJ, Thompson JF, Atkins MB, Byrd DR, Buzaid AC, Cochran AJ, Coit DG, Ding S, et al: Final version of 2009 AJCC melanoma staging and classification. J Clin Oncol 27: 6199-6206, 2009.

36. Kutikhin AG: Association of polymorphisms in TLR genes and in genes of the Toll-like receptor signaling pathway with cancer risk. Hum Immunol 72: 1095-1116, 2011.

37. Tengroth L, Millrud CR, Kvarnhammar AM,Kumlien Georén S, Latif L and Cardell LO: Functional effects of Toll-like receptor (TLR)3, 7, 9, RIG-I and MDA-5 stimulation in nasal epithelial cells. PLoS One 9: e98239, 2014.

38. Zhang Y, Yang H, Barnie PA, Yang P, Su Z, Chen J, Jiao Z, Lu L, Wang S and Xu H: The expression of Toll-like receptor 8 and its relationship with VEGF and Bcl-2 in cervical cancer. Int J Med Sci 11: 608-613, 2014.

39. Ridnour LA, Cheng RY, Switzer CH, Heinecke JL, Ambs S, Glynn S, Young HA, Trinchieri G and Wink DA: Molecular pathways: Toll-like receptors in the tumor microenvironment - poor prognosis or new therapeutic opportunity. Clin Cancer Res 19: 1340-1346, 2013.

40. Krieg AM: Antitumor applications of stimulating toll-like receptor 9 with $\mathrm{CpG}$ oligodeoxynucleotides. Curr Oncol Rep 6: 88-95, 2004.

41. Aspord C, Tramcourt L, Leloup C, et al: Imiquimod inhibits melanoma development by promoting pDC cytotoxic functions and impeding tumor vascularization. J Invest Dermatol 134: 2551-2561, 2014. 
42. Wang D, Precopio M, Lan T, Yu D, Tang JX, Kandimalla ER and Agrawal S: Antitumor activity and immune response induction of a dual agonist of Toll-like receptors 7 and 8. Mol Cancer Ther 9: 1788-1797, 2010.

43. Wang J, Kobayashi M, Han M, Choi S, Takano M, Hashino S, Tanaka J, Kondoh T, Kawamura K and Hosokawa M: MyD88 is involved in the signaling pathway for Taxol-induced apoptosis and TNF-alpha expression in human myelomonocytic cells. Br J Haematol 118: 638-645, 2002.

44. Tyring S, Conant M, Marini M, Van Der Meijden W and Washenik K: Imiquimod; an international update on therapeutic uses in dermatology. Int J Dermatol 41: 810-816, 2002.
45. Gupta AK, Cherman AM and Tyring SK: Viral and nonviral uses of imiquimod: A review. J Cutan Med Surg 8: 338-352, 2004

46. Yates CM and Sternberg MJ: The effects of non-synonymous single nucleotide polymorphisms (nsSNPs) on protein-protein interactions. J Mol Biol 425: 3949-3963, 2013.

47. Gast A, Bermejo JL, Claus R, Brandt A, Weires M, Weber A, Plass C, Sucker A, Hemminki K, Schadendorf D and Kumar R: Association of inherited variation in Toll-like receptor genes with malignant melanoma susceptibility and survival. PLoS One 6: e24370, 2011.

48. Askar E, Ramadori G and Mihm S: Toll-like receptor 7 rs179008/Gln11Leu gene variants in chronic hepatitis C virus infection. J Med Virol 82: 1859-1868, 2010. 